\title{
Influence of Social Psychological Status On Efavirenz And Nevirapine Plasma Concentration Among HIV Patients In Kenya
}

Musa Otieno Ngayo ( $\nabla$ musaotieno@yahoo.com )

Kenya Medical Research Institute

Margaret Oluka

University of Nairobi

Wallace Dimbuson Bulimo

University of Nairobi

Faith Apolot Okalebo

University of Nairobi

\section{Research Article}

Keywords: HIV stigma, disclosure and social support, adherence, efavirenz and Nevirapine plasma concentration

Posted Date: July 1st, 2021

DOI: https://doi.org/10.21203/rs.3.rs-634234/v1

License: (c) (i) This work is licensed under a Creative Commons Attribution 4.0 International License. Read Full License 


\section{Abstract}

HIV-related stigma, lack of disclosure and social support are still a hindrance to HIV testing, care, and prevention. We evaluated the influence of these socio-phycological status on nevirapine (NVP) and efavirenz (EFV) plasma concentrations among HIV patients in Kenya. Blood samples were obtained from 254 and 312 consenting HIV patients on NVP and EFV based first-line Antiretroviral therapy (ART) respectively and a detailed structured questionnaire was administered. The NVP and EFV plasma level was measured by liquid chromatography - tandem mass spectrometry (LC-MS/MS). The median duration of living with HIV infection was 5 years (IQR $=1-11$ years) and a median duration since ART initiation was 3 years (IQR $=1-8$ years). There were $68.1 \%$ and $65.4 \%$ of the patients on NVP and EFV respectively who did not feel guilty for being HIV positive. The disclosure rate was about $96.1 \%$ and $94.6 \%$ of patients on NVP and EFV respectively. About $85 \%$ and $78.2 \%$ of patients on NVP and EFV respectively who got social support as much as needed. The non-adherence to ART in the past 30 days was $64.6 \%$ and $66.3 \%$ patients on NVP and EFV respectively. The median (IQR) plasma concentration were [6237.5 ng/mL, IQR 45188-8964 ng/mL] for NVP and [2739.5 ng/mL, IQR $1878-4891.5 \mathrm{ng} / \mathrm{mL}$ ] for EFV. There were $14.2 \%$ and $4.5 \%$ patients on NVP and EFV respectively with suboptimal plasma concertation associated with poor viral suppression. Multivariate linear regression analysis showed feeling guilty for being HIV positive (adjusted $\beta=954,95 \% \mathrm{Cl}=192.7$ to $2156.6 ; p=0.014$ ) or feeling worthless for being HIV positive (adjusted $\beta=852,95 \% \mathrm{Cl}=64.3$ to 1639.7; $p=0.034$ ); being certain of telling the primary sexual partner about HIV positive status (adjusted $\beta 363,95 \% \mathrm{Cl}, 97.9$ to $628.1 ; p=0.007$ ); disclosing HIV status to neighbors (adjusted $\beta=1731,95 \% \mathrm{Cl}=376$ to $3086 ; p=0.012$ ) and getting transportation to hospital whenever needed (adjusted $\beta$ $=-1143.3,95 \% \mathrm{Cl}=-1914.3$ to $-372.4 ; p=0.004$ ) were associated with NVP/EFV plasma levels. The NVP and EFV plasma level was highly heterogenous with a significant proportion of patients reporting levels correlated with poor viral suppression. The patient's stigma, lack of disclosure and social support contributes significantly on the overall ART treatment outcome. Taking these factors into consideration, HIV treatment may be personalized to achieve optimal treatment success

\section{Introduction}

Although the current trend on global HIV epidemic has stabilized, data imply disappointingly high level of infection, an indictment of irregular control progress in countless countries [1]. The HIV pandemic continues to be the leading cause of death in sub-Saharan Africa with Kenya having the joint third-largest HIV epidemic in the world (alongside Tanzania) with 1.6 million people living with HIV [1]. HIV infection affects every breadths of human's life including physical, psychological, social and spiritual dimensions [2, 3]. In as much as HIV infection has been reported in Kenya for the last four decades, this infection is still is dreaded by many mainly due to misinformation about the disease and consequently the stigma and exclusion associated with the infection [4]. People living with HIV (PLWHA) are charged with both medical and social problems associated with the disease [5]. HIV infection among large population, results in stigma both for those infected and affected [6, 7]. Further, infection consistently results in loss of socio-economic status, employment, income, housing, health care and mobility. The outcome of stigma includes but not limited to increased secrecy (lack of disclosure) and denial, which is not only a stimulus for HIV transmission, but also a cause for poor disclosure and subsequent lack or inadequate social support $[5,7]$.

Antiretroviral therapy (ART) is an integral component in reducing the burden of HIV. Globally, at the end of $2020,67 \%$ of 38 million PLWHA were on ART [1]. Remarkable scale up of ART has put Kenya on track to reach the target on AIDSrelated deaths. At the end of 2020 , about $74 \%$ of adults and $73 \%$ of children in Kenya needing ART were essentially receiving it [1]. A remarkable fraction of these patients (68\%) had attained viral suppression (UNAIDS, 2020). At the time of this study, the first-line ART guidelines for children, youth and adults in Kenya typically contained a backbone of two 
nucleoside reverse transcriptase inhibitors (NRTIs; zidovudine [AZT], or tenofovir [TDF] with lamivudine [3TC]), plus one non-nucleoside reverse transcriptase inhibitor (NNRTI), either nevirapine (NVP) or efavirenz (EFV) [8].

Therapeutic drug exposure is a major requirement for ART management [9]. Suboptimal exposure to ART, especially NNRTIs (NVP and EFV), jeopardizes ART treatment success [10]. Generally, efavirenz and nevirapine plasma concentrations are influenced by several factors including host pharmacogenetics as well as pharmacoecological factors such as ART adherence [11]. The pharmacoecologic factors are those primarily affect adherence including lifestyle, stigma and disclosure among others. HIV stigma negatively affects ART utilization and the quality of care [5]. Social support and disclosure have been shown to significantly affect treatment outcomes in many settings [4]. Counselling and social support for both the infected and affected people is associated with effective coping with each stage of the infection and enriches the quality of life and hence adherence to ART [2]. This study, evaluated the influence HIV stigma, disclosure and social support on ART adherence and the steady-state plasma concentrations of NVP and EFV among HIV patients receiving ART in one of the largest and oldest cosmopolitan care and treatment centers in Kenya.

\section{Methods}

\section{Study design and setting}

This was a cross-sectional study conducted between August, 2016 to January 2020. Data presented in this study was part of a study that aimed at assessing the pharmacogenetic and pharmacoecologic etiology of sub-optimal responses to non-nucleoside reverse transcriptase inhibitor (NNRTI) among HIV patients in Nairobi, Kenya. Patients were recruited in this study if they were; HIV infected adults (aged above 18 years); receiving first line ART comprising of AZT or ABC, 3TC, and EFV or NVP for at least 12 months at the Family AIDS Care and Educational Services (FACES) based at Kenya Medical Research Institute (KEMRI) in Nairobi Kenya and were willing to give voluntary written informed consent. The study population and site has been described in detail in our previous publication Ngayo et al., [12]. This research was carried out in accordance with the basic principles defined in Guidance for Good Clinical Practice and the Principles enunciated in the Declaration of Helsinki (Edinburg, October 2000). This protocol and the corresponding informed consent forms used in this study were reviewed and permission obtained from, the Kenya Medical Research Institute Scientific Review Unit (SERU) (Protocol No SSC 2539). Written informed consent was obtained from all participants before enrollment.

\section{Sample size}

Sample size calculation used the formula described by Lemashow [13] based on population proportion estimation with specified relative precision. The alpha (a) was set at 0.05 , relative precision $(\varepsilon)$ at 0.20 and proportion of HIV infected individuals with sub-optimal NVP/EFV plasma levels during a 12-month ART at 15\% $(14,15)$. A total of 599 participants were recruited to achieve 0.95 power where by recruitment of patients per treatment arm was done proportionate to size yielding 269 and 330 patients on NVP and EFV respectively.

\section{Data collection}

An exhaustive structured interview was used to collect patient-related information from all the study patients. The data collected included demographic characteristics, clinical history, adherence and HIV stigma. A pilot study had been conducted to test the questionnaire and other key points in the interviews. Some of the key points explored in the structured questionnaire included stigma and segregation of people living with HIV (self-worth, guilt, emotional feeling); challenges of living with HIV, such as access to health services and community life; experiences/issues with the HIV disclosure and adherence to medications. The interviews were conducted by a clinician in a separated private room. 
The second part of the questionnaire was filled by retrospective review of patient medical records to abstract data on occurrence of any adverse drug reactions, evidence of treatment failures, adherence to clinic appointments.

Whole blood samples ( $5 \mathrm{~mL}$ ) at 12-16 hours post ARV uptake was collected using EDTA anticoagulant tubes for determining the concentration of NVP and EFV plasma concentration.

\section{Determination of nevirapine and efavirenz plasma concentrations}

The nevirapine and efavirenz plasma concentrations were measured at the laboratory using a tandem quadrupole mass spectrometer (LC/MS/MS) designed for ultra-high performance: Xevo TQ-S (Waters Corporation, U.S.A) as described by Reddy et al. [16]. Plasma samples were first subjected to a thorough in-house method for the inactivation of the HIV virus. Plasma samples were extracted using the Bond Elut C18 cartridges according to manufacturer's instructions (Agilent Technologies, USA). The Eluents were then completely evaporated using Thermo Scientific ${ }^{\mathrm{TM}}$ Reacti-Vap $^{\mathrm{TM}}$ Evaporators (Thermo Fisher Scientific Inc, USA) at $37^{\circ} \mathrm{C}$ for $30 \mathrm{~min}$. This was then reconstituted using $100 \mu \mathrm{l}$ of equal parts 1:1 acetonitrile and water, vortexed briefly and transferred into $50 \mathrm{ml}$ capped vials and placed into Xevo TQ-S (Waters Corporation, U.S.A) for quantification. About $1 \mu$ of the samples was injected automatically into the LC/MS/MS instrument and quantified within 5 minutes

\section{Data analysis}

All data were subjected to descriptive data analysis. Frequencies and percentages were used to present the sociodemographic data. HIV stigma, disclosure and social support related factors influences the drug plasma concentrations through drug adherence. The association between these variables and ART drug adherence was first evaluated using chi-square or Fishers exact test. Significant variables were then analyzed for association with NVP and EFV plasma levels. Steady-state NVP and EFV plasma concentrations were not normally distributed by the Shapiro- Wilk test, hence Kruskal-Wallis test and Dunn's test and quantile regression analysis were used to evaluate variations and association with NVP and EFV plasma levels at $5 \%$ significance level. All statistical analyses were performed using STATA v 13 (StataCorp LP, Texas, USA). The NVP plasma concentration was categorized as $<3100 \mathrm{ng} / \mathrm{mL}$ (below therapeutic range), $3100-4300 \mathrm{ng} / \mathrm{mL}$ (therapeutic range) and $>4300 \mathrm{ng} / \mathrm{mL}$ (above therapeutic range). For EFV, the concentrations of $<1000 \mathrm{ng} / \mathrm{ml}$ was considered below therapeutic range, 1000 to $4000 \mathrm{ng} / \mathrm{ml}$ considered therapeutic range and $>4000 \mathrm{ng} / \mathrm{ml}$ considered supratherapeutic level $[17,18]$.

\section{Results}

\section{Baseline characteristics of study participants}

Table 1 summarizes the baseline characteristics of study population. The results from the 254/269 (94.4\%) and $312 / 330(94.5 \%)$ response rate of patients on NVP and EFV respectively with all the relevant data were analyzed. The median age of the patients was 41 years (IQR = 35-47 years), with a median duration of living with HIV infection of five years (IQR $=1-11$ years) and a median duration since ART initiation of three years (IQR $=1-8$ years). Among these patients $342(60.4 \%)$ were female, 379 (67\%) were married, 367(64.8\%) were Bantus, $106(18.2 \%)$ had a previous partner who died. Only 3.5\% and 5.8\%; $19.7 \%$ and $17.3 \%$ (on NVP and EFV respectively) were currently smoking and taking alcohol respectively. Out of 254 patients on NVP and 312 on EFV, majority $74.4 \%$ and $73.3 \%$ stated the difficulties disclosing their HIV status. On the contrary, the majority $79.1 \%$ and $75.9 \% ; 68.1 \%$ and $65.4 \%$ (on NVP and EFV respectively) did not feel immoral or guilty for being HIV positive, respectively. Both patients on NVP and EFV, majority did not feel ashamed or worthless for being HIV positive and were very ready to tell their primary sexual partner of their HIV status. The majority $85 \%$ (NVP) and 78.2\% (EFV) got as much as would like useful advice about important things in life $(p=0.022)$. Similarly, the majority of these patients got as much as possible chance to talk to someone about 
work/household problems, about personal/family problem, people who cared about their situations and got as much love and affection. Majority of the patients also got emergency financial and transportation support but there was no significant difference by ART regimen. Other than age $(p=0.046)$ and number of life sexual partners $(p=0.019)$, there was no significant difference in the baseline characteristics between patients on NVP and those on EFV.

Table 1. Baseline characteristics of the study patients

\begin{tabular}{|c|c|c|c|c|c|c|c|c|}
\hline \multicolumn{2}{|l|}{ Variable } & \multicolumn{2}{|c|}{ All Patients ( $(n=566)$} & \multicolumn{2}{|c|}{ Nevirapine $(n=254)$} & \multicolumn{2}{|c|}{ Efavirenze $(\mathrm{n}=312)$} & \multirow[t]{2}{*}{$p$ Value } \\
\hline & & n & $(\%)$ & n & (\%) & n & (\%) & \\
\hline \multirow{5}{*}{ Age (years) } & Median (IQR) & 41 & $(35-47)$ & 42 & $(36-48)$ & 40 & $(34-47)$ & \\
\hline & $20-30$ & 66 & 11.7 & 25 & 9.8 & 41 & 13.1 & \\
\hline & $31-40$ & 210 & 37.1 & 84 & 33 & 126 & 40.4 & 0.046 \\
\hline & $41-50$ & 202 & 35.7 & 106 & 41.7 & 96 & 30.8 & \\
\hline & $>51$ & 88 & 15.5 & 39 & 15.4 & 49 & 15.7 & \\
\hline \multirow{2}{*}{ Gender } & Female & 342 & 60.4 & 163 & 64.2 & 179 & 57.4 & 0.102 \\
\hline & Male & 224 & 39.6 & 91 & 35.8 & 133 & 42.6 & \\
\hline & Married & 379 & 67 & 165 & 65.0 & 214 & 68.6 & \\
\hline & Single & 154 & 27.2 & 72 & 28.4 & 82 & 26.3 & \\
\hline Marrital status & Divorced & 26 & 4.6 & 14 & 5.5 & 12 & 3.9 & 0.703 \\
\hline & Widow & 7 & 1.2 & 3 & 1.2 & 4 & 1.3 & \\
\hline & Employed & 193 & 34.1 & 80 & 31.5 & 113 & 36.2 & \\
\hline Occupation & Unemployed & 102 & 18 & 44 & 17.3 & 58 & 18.9 & 0.354 \\
\hline & Self employed & 271 & 47.9 & 130 & 51.2 & 141 & 45.2 & \\
\hline & Bantu & 367 & 64.8 & 161 & 63.4 & 206 & 66.0 & \\
\hline Ethnicity & Nilotes & 190 & 33.6 & 91 & 35.8 & 99 & 31.7 & 0.256 \\
\hline & Cushites & 9 & 1.7 & 2 & 0.8 & 7 & 2.2 & \\
\hline & Primary & 174 & 30.7 & 69 & 27.2 & 105 & 33.4 & \\
\hline & Secondary & 203 & 35.9 & 102 & 40.2 & 101 & 32.4 & 0.17 \\
\hline Education level & Tertiary & 182 & 32.2 & 81 & 31.9 & 101 & 32.4 & \\
\hline & Non-formal & 7 & 1.2 & 2 & 0.8 & 5 & 1.6 & \\
\hline Cigarette smoking & Yes & 27 & 4.8 & 9 & 3.5 & 18 & 5.8 & 0.24 \\
\hline Cigarette smoking & No & 539 & 95.2 & 245 & 96.5 & 294 & 94.3 & \\
\hline & Yes & 104 & 18.4 & 50 & 19.7 & 54 & 17.3 & 0.099 \\
\hline Alcohol consumption & No & 462 & 81.6 & 204 & 80.3 & 258 & 82.7 & \\
\hline & Median (IQR) & 18 & $(17-20)$ & 18 & $(17-19)$ & 18 & $(17-20)$ & \\
\hline Age of sexual debut (Years) & $<18$ & 371 & 65.6 & 166 & 65.4 & 205 & 65.7 & 0.929 \\
\hline & $>18$ & 195 & 34.5 & 88 & 34.7 & 107 & 34.3 & \\
\hline & Median (IQR) & 2 & $(1-5)$ & 2 & $(1-4)$ & 3 & $(1-5)$ & \\
\hline Sufotimn nenal nartnem & None & 3 & 0.5 & 2 & 0.8 & 1 & 0.3 & \\
\hline Lifetime sexual partners & 1 & 214 & 37.8 & 110 & 43.3 & 104 & 33.3 & 0.019 \\
\hline & $>1$ & 349 & 61.7 & 142 & 55.9 & 207 & 66.4 & \\
\hline & 3TC, ABC, EFV & 1 & 02 & 0 & 0 & 1 & 03 & \\
\hline & 3TC, TDF, EFV & 187 & 33.1 & 0 & 0 & 187 & 59.9 & \\
\hline & ЗTC, ZDV, EFV & 124 & 21.9 & 0 & 0 & 124 & 39.7 & \\
\hline Curreat ART rezimen & YTC, ABC, NVP & 1 & 0.2 & 1 & 0.4 & 0 & 0 & 0.0001 \\
\hline & 3TC, TDF, NVP & 159 & 28.1 & 159 & 62.6 & 0 & 0 & \\
\hline & 3TC, ZDV, NVP & 93 & 164 & 93 & 36.6 & 0 & 0 & \\
\hline & 3TC, dAT, NVP & 1 & 0.2 & 1 & 0.4 & 0 & 0 & \\
\hline Difficult to tell others about my & Agree & 418 & 73.8 & 189 & 74.4 & 229 & 73.4 & 0.848 \\
\hline HIV infection & Disagree & 148 & 26.2 & 65 & 25.6 & 883 & 26.6 & \\
\hline Feeling guilty for being HIV & Agree & 189 & 33.4 & 81 & 31.9 & 108 & 34.6 & 0.531 \\
\hline positive & Disagree & 377 & 66.6 & 173 & 68.1 & 204 & 65.4 & \\
\hline Feeling worthless for being HIV & Agree & 137 & 24.2 & 55 & 21.7 & 82 & 26.3 & 0.236 \\
\hline 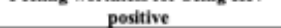 & Disagree & 429 & 75.8 & 199 & 78.4 & 230 & 73.7 & \\
\hline 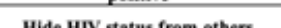 & Agree & 403 & 71.2 & 186 & 73.2 & 217 & 69.5 & 9.352 \\
\hline Hide HIV status from others & Disagree & 163 & 28.8 & 68 & 26.8 & 95 & 30.5 & \\
\hline 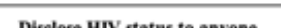 & Yes & 539 & 95.2 & 244 & 96.1 & 295 & 94.6 & 0.435 \\
\hline Disclose HIV status to anyone & No & 27 & 4.7 & 10 & 3.9 & 17 & 5.4 & \\
\hline & Yes & 446 & 78.8 & 204 & 80.3 & 242 & 77.8 & \\
\hline Disclosed HIV status to partner & No & 63 & 11.1 & 25 & 9.8 & 38 & 12.2 & 0.065 \\
\hline & Not applicable & 57 & 10.1 & 25 & 9.8 & 32 & 10.3 & \\
\hline & Yes & 349 & 61.7 & 106 & 65.4 & 183 & 58.7 & \\
\hline Disclosed HIV status to family & No & 212 & 37.5 & 87 & 34.4 & 125 & 40.1 & 0.178 \\
\hline & Not applicable & 5 & 0.9 & 1 & 0.4 & 4 & 1.3 & \\
\hline & Yes & 12 & 2.1 & 5 & 1.9 & 7 & 2.2 & \\
\hline Disclosed HIV status to the public & No & 513 & 90.6 & 231 & 90.4 & 282 & 90.4 & 0.965 \\
\hline & Not applicable & 41 & 7.2 & 18 & 7.1 & 23 & 7.4 & \\
\hline & As much as I wowad like & 460 & 81.3 & 216 & 85.0 & 244 & 78.2 & \\
\hline Get useful advice abeat important & Less then I worild like & 79 & 139 & 33 & 12.9 & 46 & 14.7 & \\
\hline $\begin{array}{l}\text { things in life } \\
\text {. }\end{array}$ & Much less then I would like & "1 & 1.9 & 1 & 0.4 & 10 & 32 & 0.022 \\
\hline & Never & 16 & 28 & 4 & 1.6 & 12 & 3.9 & \\
\hline & As much as I woend like & 337 & 59.5 & 162 & 63.8 & 175 & 56.1 & \\
\hline & Less then I woridd like & 92 & 163 & 40 & 15.8 & 52 & 16.7 & \\
\hline Get finascial help during emergency & Much less then I worild tike & 44 & 7.8 & 12 & 4.7 & 32 & 10.3 & 0.066 \\
\hline & Never & 93 & 164 & 40 & 15.8 & $\$ 3$ & 16.9 & \\
\hline & As much as I woond like & 357 & 63.1 & 169 & 66.5 & 188 & 603 & \\
\hline 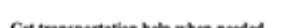 & Less then I wowild like & 81 & 143 & 38 & 14.9 & 43 & 138 & \\
\hline Get transportatios help whee needed & Much less then I wouild like & 45 & 79 & 15 & 5.9 & 30 & 9.6 & 0.19 \\
\hline & Never & 83 & 14.7 & 32 & 12.6 & $\$ 1$ & 16.4 & \\
\hline & As much as I woend like & 456 & $\$ 0.6$ & 212 & 83.5 & 244 & 78.2 & \\
\hline 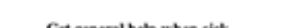 & Less then I wovild like & 67 & 11.8 & 27 & 10.6 & 40 & 128 & \\
\hline Get geatral help whes sick & Much less then I would like & 18 & 3.2 & 6 & 2.4 & 12 & 3.9 & 0.437 \\
\hline & Never & 25 & 4.4 & 9 & 3.5 & 16 & 52 & \\
\hline
\end{tabular}

\section{ART adherence}

In this study non-adherence was defined as missing to take current ART or missing to take ART dose in the past 30 days. Among all the study patients $371(n=566 ; 65.6 \%) ; 164(n=254 ; 64.6 \%)$ on NVP and $207(n=312 ; 66.3 \%)$ on EFV reported non-adherence in the last 30 days (Fig. 1). 


\section{Efavirenz and Nevirapine plasma concentration}

Among the patients on nevirapine based ART regimen, the majority (54.3\%) had plasma levels of $>6000 \mathrm{ng} / \mathrm{ml}$ considered levels for durable viral suppression. There were 80 (31.5\%) patients with NVP levels between 3400 to $6000 \mathrm{ng} / \mathrm{ml}$ considered levels for viral mutant selection windows and the least $36(14.2 \%)$ who had NVP plasma concertation of $<3400 \mathrm{ng} / \mathrm{ml}$ considered levels for poor viral suppression $(p<0.05)$. For patients on efavirenz based ART regimen, the majority (63.8\%) had plasma concentrations between 1000 to $4000 \mathrm{ng} / \mathrm{ml}$ considered levels for viral mutant selection windows with some $14(4.5 \%)$ had plasma concentrations of $<1000 \mathrm{ng} / \mathrm{ml}$ considered levels for poor viral suppression window $(p<0.05)$.

\section{Relationship between HIV related stigma, disclosure and social support and ART adherence}

HIV stigma, disclosure and social support related factors influence the drug plasma level by affecting drug adherence. The study first assessed the relationship between social physiological status variables with ART drug adherence and only those factors found significant were then evaluated against drug plasma level. Among these factors included, gender, age, substance abuse, age of sexual debut, number of sexual life partners. Among HIV stigma related factors assessed included; feeling guilty for being HIV positive, hiding HIV status from others and feeling certain to tell primary sexual partner about HIV status. HIV disclose related factors included being able to disclose HIV status to anyone and disclosing the HIV status to family members. Among HIV social support related factors assessed included; getting useful advice about important things in life, getting chance to talk to someone about work or household problems, getting love and affection, being helped with household duties, getting financial and transportation help. Nutritional related factors included: Regular access to staple food, regular uptake of porridge, access to food supplements, Food intake in the last 5-10 days, presence of wasting and feeding syndrome risk (Table 2).

Table 2. Relationship between HIV stigma, disclosure and social support and ART drug adherence 


\begin{tabular}{|c|c|c|}
\hline \multirow[b]{2}{*}{ Variab le } & \multicolumn{2}{|c|}{$\begin{array}{l}\text { FISHER'S EXACT TEST } \\
\text { ART d rug Adherence }\end{array}$} \\
\hline & $\begin{array}{l}\text { Ner irapine } \\
P \text { value }\end{array}$ & $\begin{array}{c}\text { E favirenz } \\
P \text { value }\end{array}$ \\
\hline \multicolumn{3}{|l|}{ Socio-dem ographic variables } \\
\hline Gender & 0.318 & 0.253 \\
\hline Age & 0.393 & 0.129 \\
\hline Marital status & 0.06 & 0.368 \\
\hline Occupation & 0.952 & 0.565 \\
\hline Religion & 0.785 & 0.689 \\
\hline Education & 0.611 & 0.124 \\
\hline Vacational schooling & 0.482 & 0.209 \\
\hline L iving with partner & 0.363 & 0.871 \\
\hline Had more than one partner & 0.97 & 0.533 \\
\hline Previous partner died & 0.919 & 0.953 \\
\hline Smoking & 0.725 & 0.798 \\
\hline Alcohol use number of times & 0.011 & 0.011 \\
\hline Age of sexual debut & 0.028 & 0.024 \\
\hline Number of sexual life partners & 0.0001 & 0.0001 \\
\hline \multicolumn{3}{|l|}{ HIV stigma related factors } \\
\hline Difficult to tell others about my HIV infection & 0.234 & 0.281 \\
\hline Being HIV positive $m$ akes me feel imm oral & 0.260 & 0.005 \\
\hline Being HIV positive makes $m e$ feel guilty & 0.035 & 0.314 \\
\hline Being HIV positive $m$ akes me feel ashamed & 0.570 & 0.794 \\
\hline Being HIV positive makes me feel it worthless & 0.750 & 0.344 \\
\hline Being HIV positive makes me feel it is my own fault & 0.111 & 0.318 \\
\hline Hide HIV status from others & 0.005 & 0.605 \\
\hline \multicolumn{2}{|l|}{ HIV disclosure related factors } & 0.0001 \\
\hline Disclose HIV status to anyone & 0.332 & 0.033 \\
\hline Disclosed HIV status to partner or spouse & 0.197 & 0.578 \\
\hline Disclosed HIV status to family members & 0.570 & 0.730 \\
\hline Disclosed HIV status to friends & 0.908 & 0.383 \\
\hline Disclosed HIV status to neighbor & 0.306 & 0.202 \\
\hline Disclosed HIV status to employers & 0.217 & 0.579 \\
\hline Disclosed HIV status to religious leaders & 0.362 & 0.582 \\
\hline Disclosed HIV status to the public & 0.748 & 0.331 \\
\hline $\begin{array}{l}\text { Number disclosed about HIV status in the family } \\
\text { HIV social supp ort }\end{array}$ & 0.185 & 0.055 \\
\hline Get useful advice about im portant things in life & 0.022 & 0.005 \\
\hline $\begin{array}{l}\text { G et chance to talk to som eone about work or household } \\
\text { problems }\end{array}$ & 0.005 & 0.001 \\
\hline $\mathrm{G}$ et chance to talk to som eone about per sonal or family & 0.071 & 0.002 \\
\hline I have people who cares about what happens to me & 0.256 & 0.038 \\
\hline I get love and affection & 0.0001 & 0.008 \\
\hline Help with household duties & 0.007 & 0.001 \\
\hline Get financial help during emergency & 0.005 & 0.045 \\
\hline Get transportation help when needed & 0.001 & 0.014 \\
\hline G et general help when sick & 0.138 & 0.009 \\
\hline
\end{tabular}

\section{Variation in efavirenz and nevirapine plasma level HIV stigma, disclosure and social support related variables}

Table 3 summarizes the variation in the median NVP and EFV plasma concentration and socio-demographic, sexual behavior, HIV stigma and disclosure characteristic. Patients who disclosed their HIV status to their employer had higher median (IQR) EFV plasma concentration $(3157, \mathrm{IQR}=2001-5976 \mathrm{ng} / \mathrm{mL})$ than those that did not $(2173.5$, IQR $=$ $1655.5-3208.5 \mathrm{ng} / \mathrm{mL} ; p=0.041)$. Patients who did not disclose their HIV status to religious leaders had higher median (IQR) EFV plasma concentration $(2821.5, \mathrm{IQR}=1945-5270 \mathrm{ng} / \mathrm{mL})$ than those who did $(1998.5, \mathrm{IQR}=1548-2520$ $\mathrm{ng} / \mathrm{mL} ; p=0.0031)$. Further, patients who disclosed their HIV status to the public had higher median (IQR) EFV plasma concentration $(3097, \mathrm{IQR}=2872-5976 \mathrm{ng} / \mathrm{mL})$ as compared to patients who did not $(1965, \mathrm{IQR}=1639-2763 \mathrm{ng} / \mathrm{mL} ; p$ $=0.0117)$.

Table 3. Variation in median efavirenz and nevirapine plasma concentration and HIV stigma, disclosure and social support related variables 


\begin{tabular}{|c|c|c|c|c|c|c|c|c|c|c|}
\hline \multirow[t]{2}{*}{ Variable } & \multicolumn{5}{|c|}{ NEVIRAPINE $(N=254)$} & \multicolumn{5}{|c|}{ EFAVIRENZ $(\mathrm{N}=312)$} \\
\hline & \multirow{3}{*}{$\frac{\mathrm{n}}{25}$} & Median & \multicolumn{2}{|c|}{$(\mathrm{IQR})$} & $\mathrm{p}$ & $\mathrm{n}$ & Median & \multicolumn{2}{|c|}{ (IQR) } & \multirow[t]{2}{*}{$\mathrm{p}$} \\
\hline & & & & & & & & & & \\
\hline $20-30$ & & 6034 & 4448 & 7817 & & 41 & 2961 & 1679 & 4603 & \\
\hline $31-40$ & 84 & 6207 & 4558 & 8946.5 & 0.667 & 126 & 2698.5 & 1918 & 5976 & 0.476 \\
\hline $41-50$ & 106 & 6368 & 4599 & 9784 & & 96 & 26855 & 1950.5 & 4282.5 & \\
\hline$>51$ & 39 & 6011 & 4518 & 8843 & & 49 & 2754 & 1833 & 4074 & \\
\hline \multicolumn{11}{|l|}{ Gender } \\
\hline Male & 91 & 5917 & 4449 & 8638 & 0.387 & 133 & 2747 & 1918 & 5336 & 0.728 \\
\hline Female & 163 & 6364 & 4558 & 9293 & & 179 & 2712 & 1868 & 4647 & \\
\hline \multicolumn{11}{|l|}{ Vacational schooling } \\
\hline Yes & 111 & 6699 & 5140 & 9906 & 0.031 & 146 & 2645.5 & 1917 & 4713 & 0.423 \\
\hline No & 143 & 5773 & 4117 & 8729 & & 166 & 2905 & 1868 & 5492 & \\
\hline Alcohol use number of times & & & & & & & & & & \\
\hline Never & 204 & 6351 & 4407 & 9194 & & 258 & 2636 & 1857 & 4744 & \\
\hline 1 time & 33 & 6180 & 5128 & 8573 & 0.758 & 45 & 3093 & 2068 & 5787 & 0.373 \\
\hline$\geq 2$ times & 17 & 5773 & 4935 & 8034 & & 9 & 2747 & 2086 & 5988 & \\
\hline Being HIV positive malies me & & & & & & & & & & \\
\hline feel guilty & & & & & & & & & & \\
\hline Agree & 81 & 5557 & 4247 & 7633 & 0.016 & 108 & 2645.5 & 1895 & 5171.5 & 0.927 \\
\hline Disagres & 173 & 6511 & 4607 & 9863 & & 204 & 2854 & 1869 & 4839.5 & \\
\hline Being HIV positive malies me & & & & & & & & & & \\
\hline feel it worthless & & & & & & & & & & \\
\hline Agree & 55 & 5243 & 3975 & 7311 & 0.054 & 82 & 2756 & 1951 & 4319 & 0.837 \\
\hline Disagree & 199 & 6511 & 4599 & 9755 & & 230 & 2720.5 & 1838 & 5204 & \\
\hline Disclosed HIV status to anyon & & & & & & & & & & \\
\hline Yes & 204 & 6402.5 & 4564.5 & 9180.5 & & 242 & 2759.5 & 1886 & 5204 & \\
\hline No & 25 & 4853 & 3450 & 6202 & 0.036 & 38 & 2091 & 1918 & 5336 & 0.565 \\
\hline Not applicable & 25 & 6273 & 4577 & 9909 & & 32 & 2556 & 1750 & 3488 & \\
\hline Disclosed HIV status to anyon & & & & & & & & & & \\
\hline $\begin{array}{c}\text { to finmily members } \\
\text { Yes }\end{array}$ & 166 & 5967.5 & 4444 & 7966 & & 183 & 2592 & 1917 & 5044 & \\
\hline No & 87 & 6868 & 4951 & 10635 & 0.064 & 125 & 2867 & 1870 & 4911 & 0.312 \\
\hline Not apolicable & 1 & 8034 & 8034 & 8034 & & 4 & 1699.5 & 456.5 & 3118.5 & \\
\hline $\begin{array}{l}\text { Disclosed HIV status to anyon } \\
\text { to neighbor }\end{array}$ & & & & & & & & & & \\
\hline Yes & 13 & 5239 & 3631 & 7009 & & 22 & 3079 & 1917 & 7572 & \\
\hline No & 234 & 6237.5 & 4558 & 9095 & 0.210 & 280 & 2739.5 & 1902 & 4837 & 0.088 \\
\hline Not apolicable & 7 & 7966 & 6372 & 9909 & & 10 & 2027.5 & 857 & 2961 & \\
\hline $\begin{array}{l}\text { Disclosed HIV status to anyon } \\
\text { to religious leaders }\end{array}$ & & & & & & & & & & \\
\hline Yes & 18 & 4479 & 2960 & 7009 & & 26 & 2440 & 1633 & 5909 & \\
\hline No & 222 & 6317 & 4607 & 9293 & 0.065 & 264 & 2821.5 & 1945 & 5270 & 0.003 \\
\hline Not applicable & 14 & 6371.5 & 4211 & 8034 & & 22 & 1998.5 & 1548 & 2520 & \\
\hline Disclosed HIV status to anyon & & & & & & & & & & \\
\hline to public & & & & & & & & & & \\
\hline Yes & 5 & 5736 & 5239 & 7009 & & 7 & 3097 & 2872 & 5976 & \\
\hline No & 231 & 6202 & 4503 & 9163 & 0.869 & 282 & 2766.5 & 1918 & 5139 & 0.012 \\
\hline Not apolicable & 18 & 6595.5 & 4558 & 8034 & & 23 & 1965 & 1639 & 2763 & \\
\hline $\begin{array}{l}\text { Get financial help during } \\
\text { emergency }\end{array}$ & & & & & & & & & & \\
\hline As much as I wouild like & 162 & 6365.5 & 4558 & 8964 & & 175 & 2836 & 1918 & 4911 & \\
\hline Less than I wovild 1ike & 40 & 5468.5 & 4275 & 8191.5 & 0.492 & 52 & 2309.5 & 1789.5 & 5038 & 0.797 \\
\hline Much less than I wovild like & 12 & 7275.5 & 6056.5 & 9583 & & 32 & 2747.5 & 1615.5 & 8797.5 & \\
\hline Never & 40 & 5710.5 & 4046 & 9867.5 & & 53 & 2872 & 2043 & 4241 & \\
\hline Get transportation help when & & & & & & & & & & \\
\hline needed & & & & & & & & & & \\
\hline As much as I wouild like & 169 & 6538 & 4571 & 9198 & & 188 & 2821.5 & 1895 & 5223.5 & \\
\hline Less than I wouild 1ike & 38 & 5527.5 & 4336 & 8382 & 0.550 & 43 & 2462 & 1818 & 4872 & 0.917 \\
\hline Much less than I wovild like & 15 & 6202 & 4180 & 6868 & & 30 & 2670 & 1679 & 6875 & \\
\hline Never & 32 & 5635.5 & 3955.5 & 8750 & & 51 & 2786 & 1942 & 3875 & \\
\hline Get a neral help when sick & & & & & & & & & & \\
\hline As much as I wouild like & 212 & 6351 & 4448.5 & 9129 & & 244 & 2796.5 & 1895 & 4977.5 & \\
\hline Less than I wovild livke & 27 & 5911 & 4990 & 9411 & 0.970 & 40 & 2569 & 1999.5 & 5589.5 & 0.534 \\
\hline Much less than I wouild tike & 6 & 7039 & 5729 & 8405 & & 12 & 2447.5 & 911 & 4693 & \\
\hline Never & 9 & 5692 & 5457 & 7009 & & 16 & 2931.5 & 1613.5 & 4168 & \\
\hline
\end{tabular}

Patients with higher median (IQR) EFV plasma concentration were those who did not feel guilty for being HIV positive $(6511, \mathrm{IQR}=4607-9863 \mathrm{ng} / \mathrm{mL})$ as compared to patients who felt guilty $(5557, \mathrm{IQR}=4247-7633 \mathrm{ng} / \mathrm{mL} ; p=0.0163)$. Patients who disclosed their HIV status to their spouse $(6402.5, \mathrm{IQR}=4564.5-9180.5 \mathrm{ng} / \mathrm{mL}$ ) had higher median (IQR) NVP plasma concentration than those who did not $(4853, \mathrm{IQR}=3450-6202 \mathrm{ng} / \mathrm{mL} ; p=0.0362)$.

\section{Factors associated with drug plasma concentration}

\section{Stigma}

In multivariate linear regression analysis, feeling guilty for being HIV positive (adjusted $\beta=954,95 \% \mathrm{Cl}=192.7$ to 2156.6; $p=0.014$ ) or feeling worthless for being HIV positive (adjusted $\beta=852,95 \% \mathrm{Cl}=64.3$ to $1639.7 ; p=0.034$ ) were independent factors associate NVP plasma concentrations. For patients on EFV, being certain of telling the primary 
sexual partner about HIV positive status was associated with increased EFV plasma levels (adjusted $\beta 363,95 \% \mathrm{Cl}, 97.9$ to $628.1 ; p=0.007$ ) (Table 4).

Table 4. Regression analysis between NVP and EFV plasma concentrations and HIV stigma variables

\begin{tabular}{|c|c|c|c|c|c|c|c|c|}
\hline \multirow[b]{2}{*}{ Variable } & \multicolumn{4}{|c|}{ NEVIRAPINE $(N=254)$} & \multicolumn{4}{|c|}{ EFAVIRENZ $(\mathrm{N}=312)$} \\
\hline & Unadjusted $\beta$ & \multicolumn{2}{|c|}{$(95 \% \mathrm{CI})$} & \multirow{2}{*}{$\frac{p \text { value }}{0.307}$} & \multirow{2}{*}{$\frac{\text { Unadustad } \mathrm{B}}{-13.7}$} & \multicolumn{2}{|c|}{$(95 \% \mathrm{CI})$} & \multirow{2}{*}{$\frac{\text { prahie }}{0.284}$} \\
\hline Age & -14 & -562 & 282 & & & -38.7 & 11.4 & \\
\hline Gender & 447 & -545.5 & 1439.5 & 0.376 & -35 & -536.5 & 466.5 & 0.891 \\
\hline Vacational schooling & -926 & -17532 & -98.8 & 0.028 & 276 & -262.4 & 814.4 & 0.314 \\
\hline Alcohol use number of times & -198 & -680.7 & 284.7 & 0.42 & 330 & -534.4 & 1194.4 & 0.453 \\
\hline Age of sexual debut & -364 & -1385.8 & 657.8 & 0.484 & 54 & -459.7 & 567.7 & 0.836 \\
\hline Number of sexuallife partners & -600 & -1285.7 & 85.7 & 0.086 & -557 & -918.0 & -196.0 & 0.003 \\
\hline Number of sexual acts in the past 3 months & -46.5 & -748.9 & 6559 & 0.896 & -106 & -6489 & 436.9 & 0.701 \\
\hline Difficult to tell ot hers about my HIV infection & 141 & -958.8 & 1240.8 & 0.801 & -126 & -703.9 & 451.9 & 0.668 \\
\hline Being HIV positive makes me feel guilty & 954 & 26.7 & 1881.3 & 0.044 & 210 & -2813 & 701.3 & 0.401 \\
\hline Being HIV pos itive malses me feel it w ort hless & 1268 & 379.4 & 2156.6 & 0.005 & -33 & -744.7 & 678.7 & 0.927 \\
\hline $\begin{array}{l}\text { Feel certain to tell primary sexual partner being HIV } \\
\text { positive }\end{array}$ & 372 & -453.2 & 1197.2 & 0.376 & 426 & 243 & 827.7 & 0.038 \\
\hline Dis close HIV status to anyone & -539 & -1578.8 & 500.8 & 0.308 & 983 & -1058.0 & 3024.0 & 0.344 \\
\hline Disclosed HIV status to anyone to family members & 1051.5 & -541.5 & 2644.5 & 0.195 & 134 & -381.8 & 649.8 & 0.61 \\
\hline Disclosed HIV status to anyone to neighbor & 1675 & 137.5 & 3212.5 & 0.033 & -445 & -1441.0 & 551.0 & 0.38 \\
\hline Disclosed HIV status to amyone to employers & -112 & -12033 & 9793 & 0.84 & -489 & -10372 & 592 & 0.08 \\
\hline Disclosed HIV status to anyone to religious leaders & 1609 & -98.7 & 3316.7 & 0.065 & -410 & -9079 & 87.9 & 0.106 \\
\hline Get useful advice about importat things in life & -539 & -1778.4 & 1303.7 & 0.762 & -1343 & -483.8 & 215.1 & 0.45 \\
\hline Get financialhelp during emergency & -124.7 & -541.9 & 292.6 & 0.557 & 18.7 & -189.8 & 227.1 & 0.86 \\
\hline Get transport ation help when needed & -300 & -512.1 & -87.9 & 0.006 & -5 & -177.6 & 167.6 & 0.955 \\
\hline Get general help when sic lk & -217 & -599.7 & 165.7 & 0.265 & -158 & -562.3 & 246.3 & 0.442 \\
\hline \multirow[t]{2}{*}{ Variable } & \multicolumn{4}{|c|}{ NEVIRAPINE $(N=254)$} & \multicolumn{4}{|c|}{ EFAVIRENZ $(\mathrm{N}=312)$} \\
\hline & Adjusted $\beta$ & $(95$ & CD) & p value & Adjustad $\beta$ & $(95$ & C) & prahie \\
\hline Age & 0.421 & -71.7 & 72.5 & 0.991 & -15.5 & -52.5 & 21.6 & 0.412 \\
\hline Gender & 172 & -1010.5 & 1354.5 & 0.775 & -40.4 & -832.7 & 751.9 & 0.92 \\
\hline Vacational schooling & -1029 & 2068.4 & 10.4 & 0.042 & 306.2 & -2839 & 896.3 & 0.308 \\
\hline Alcohol use number of times & -162.5 & -811 & 486 & 0.622 & 398 & -4312 & 12272 & 0.346 \\
\hline Age of sexual debut & -1008.1 & -2745.4 & 729.1 & 0.254 & 563.5 & -424.6 & 1551.6 & 0.263 \\
\hline Number of sexuallife partners & -988 & -2156.8 & 180.8 & 0.097 & -845.7 & -1315.0 & -376.4 & 0.0001 \\
\hline Number of sexual acts in the past 3 monfhs & -2180.8 & -5358.2 & 996.6 & 0.178 & 487.3 & -32242 & 4198.8 & 0.796 \\
\hline Difficult to tell ot hers about my HIV infection & -528.5 & -16339 & 5769 & 0.347 & -177 & -10213 & 667.3 & 0.68 \\
\hline Being HIV positve malkes me feel guility & 954 & 192.7 & 1715.3 & 0.014 & 347.7 & -153.4 & 848.7 & 0.173 \\
\hline Being HIV positve malkes me feel it worthless & 852 & 64.3 & 1639.7 & 0.034 & -1433 & -7592 & 472.5 & 0.647 \\
\hline $\begin{array}{l}\text { Feel certain to tell primary sexual partmer being HIV } \\
\text { posit ive }\end{array}$ & 341.5 & -1357.0 & 2040.0 & 0.692 & 363 & 979 & 628.1 & 0.007 \\
\hline Dis close HIV status to anyone & -1042.9 & -2597.4 & 511.6 & 0.188 & 1342 & 1653.6 & 4337.6 & 0.379 \\
\hline Disclosed HIV status to anyone to family members & 812.9 & -483.3 & 2109.1 & 0.218 & 245 & -365.8 & 855.8 & 0.431 \\
\hline Disclosed HIV st atus to anyone to neighbor & 1731 & 376.0 & 3086.0 & 0.012 & -251 & -1714.1 & 1212.1 & 0.736 \\
\hline Disclosed HIV status to anyone to employers & -393.5 & -1586.1 & 799.1 & 0.516 & -505 & -14103 & 400.3 & 0.273 \\
\hline Disclosed HIV status to anyone to religious leaders & 241.6 & -1675.6 & 2158.7 & 0.804 & 29 & -11203 & 11783 & 0.96 \\
\hline Get useful advice about importat things in life & -112.7 & -1430.0 & 1204.6 & 0.866 & 16.4 & -400.5 & 433.4 & 0.938 \\
\hline Help with household duties & -3152 & -1460.0 & 829.6 & 0.588 & -26.4 & -556.1 & 103.4 & 0.178 \\
\hline Get financialhelp during emergency & 779.3 & -291.9 & 1850.6 & 0.153 & 245.0 & -304.7 & 794.7 & 0.381 \\
\hline Get transport ation help when needed & -1143.3 & -19143 & -372.4 & 0.004 & -6.6 & -377.8 & 364.7 & 0.972 \\
\hline Get general help when sic k & 212.3 & -560.5 & 985.1 & 0.589 & 74.1 & -4783 & 626.5 & 0.792 \\
\hline
\end{tabular}

\section{Disclosure}

In multivariate linear regression analysis, disclosing patient's HIV status to neighbors (adjusted $\beta=1731,95 \% \mathrm{Cl}=376$ to $3086 ; p=0.012$ ) was associated with increased NVP plasma concentrations. None of the HIV disclosure related factors were associated with EFV plasma concentrations (Table 4).

\section{Social support}

In multivariate linear regression analysis getting transportation to hospital whenever needed (adjusted $\beta=$ $-1143.3,95 \% \mathrm{Cl}=-1914.3$ to $-372.4 ; p=0.004)$ was associated with lower NVP plasma concentrations. None of the HIV social support related factors were found associated with EFV plasma concentrations (Table 4).

\section{Discussion}


Every blueprint and policies geared towards individualization of ART treatment aimed at prolonging life of HIV patients contributes significantly to the components of HIV treatment programs in many countries including Kenya. The recommendation by WHO requiring test and treat of all HIV positive patients regardless of their CD4 or viral load [19], must also appreciate that optimal ART outcomes requires an in-depth understanding of the individual's variation in response to ART, both efficacy and toxicity. ART treatment outcomes are not only influenced by patients pharmacogenetical and pharmacoecologic factors [20], social psychological (defined as human behavior as a result of the relation between mental state and social situation) well-being of patients is equally important. Stigma, disclosure and social support are social psychological - mental representations are important influence of our interactions with others and environment. This is among the first study to evaluate the influence of HIV stigma (a mark of disgrace, discounting, discrediting and discriminating associated with HIV infection and ARV use) [21], HIV disclosure (action of making new or secret of being HIV positive known) and HIV social support (the perception and actuality that one is cared for or having assistance available from other people) on the steady-state plasma concentrations of nevirapine and efavirenz among HIV patients receiving treatment in Nairobi Kenya.

HIV stigma, disclosure and availability of social support are key determinant to patient's behavior and influences adherence to HIV care, treatment and prevention. Previously in Kenya, involvement in community support networks considerably enriched adherence and treatment outcome[22]. Further, patients vigorously partaking in community support networks inclined towards attaining peak NVP plasma concentration early hours post-dosing, which were markedly higher than seen in patients not actively involved in community support networks. Countless studies have interconnected social support to better medication adherence and better clinical outcomes [23].

HIV associated stigma related factors such as feeling guilty, worthless for being HIV positive were associated with higher median NVP plasma levels. For patients on EFV based regimen, those who were certain to reveal their HIV status to their primary sexual partner had better ART adherence accompanied by higher median EFV plasma levels. Stigma and discrimination remain the paramount challenge confronted by People Living with HIV/AIDS [24]. Although data are skewed on the association between HIV stigma and NNRTI plasma level, stigma and discrimination negatively affect people living with HIV [25]. HIV-related stigma is wide-ranging and worldwide social phenomenon that exhibits within multiple social spheres, including healthcare encompassing denial of care or treatment, HIV testing without consent, confidentiality breaches, negative attitudes and humiliating practices by health workers [26]. Studies have shown an association between HIV stigma with poorer physical and mental health outcomes [27]. Stigma has also been linked with secondary health-related factors including seeking healthcare and adherence to antiretroviral therapy, and access to and usage of health and social services $[27,28]$. Inevitably, these negative outcomes of stigma are bound to affect the overall treatment outcomes in terms of therapeutic monitoring.

HIV status disclosure in our study was associated with ART adherence which was marked by higher median NVP and EFV plasma concentration regardless whether the disclosure was to a spouse, family member, religious leaders and employers. In Thailand, Sirikum et al., [29] reported no significant difference in the median ART adherence by pill count, CD4 count, or HIV viral load between HIV patient disclosed their status compared to those who did not. Studies have shown HIV disclosure to have two possible treatment outcomes [30]. On one hand; HIV status disclosure to sexual partners is a vital prevention target underlined by both WHO and the Centers for Disease Control and Prevention (CDC) [31], at an individual level and to the general public, HIV disclosure is accompanied by numeral benefits [29]. HIV infection disclosure to sexual partners is associated with less anxiety and increased social support especially among women [30, 31]. Further, HIV status disclosure is accompanied by improved access to HIV prevention and treatment programs, increased opportunities for risk reduction and increased opportunities to plan for the future. Disclosure of HIV status also expands the awareness of HIV risk to untested partners leading to better acceptance and utilization of voluntary HIV testing and counselling and changes in HIV risk behaviors [30, 31]. In addition, disclosure of HIV status to 
sexual partners empowers couples to make educated reproductive health choices that may eventually lower the number of unintended pregnancies among HIV-positive women [30]. Along with these benefits, however, there are a number of potential risks from disclosure for HIV-infected women, including loss of economic support, blame, abandonment, physical and emotional abuse, discrimination and disruption of family relationships [30, 31]. These risks may lead women to choose not to share their HIV test results with their friends, family and sexual partners. This, in turn, leads to lost opportunities for the prevention of new infections and for the ability of patients especially women to access appropriate treatment, care and support services where they are available [30, 31].

In our study, patients who had adequate social support such as getting useful advice about important things in life, having a chance to talk to someone about work, household, personal or family problems, getting love and affection had higher median NVP and EFZ plasma concentration. In South Africa, Brittain et al., [32] showed correlation between social support and stigma influencing whether or not the development of depressive symptoms. The importance of community support networks in enhancing social relationships demystifying HIV-associated stigma is well documented $[33,34]$. Evidence shows the positive effects of social support and protection on other HIV related outcomes, such as sexual risk behaviors [35,36], mental health distress and family relationships [37, 38]. Growing evidence of associations between social protection and HIV-risk reduction [39] is reflected in a number of policy documents by UNICEF, UNAIDS and PEPFAR-USAID that focus on pediatric and adolescent HIV-prevention [40, 41].

\section{Conclusions}

Some of the important limitation worth mentioning in this study included. First, the use of NVP-based ART regimen in Kenya and other countries, especially developed countries, has been considerably reduced in the recent past, meaning that this study could be relevant to a restricted number of patients. Second, standardized tools for measuring stigma, disclosure and social support were not used in this study limiting the generalizability of this study outcome. Fourth, this was a cross-sectional study, which only permitted the description of the relationship between the three sociopsychological factors and NVP/EFV plasma concentrations and not a causal conclusion. Such outcomes can be confirmed in a longitudinal study.

These limitations notwithstanding, our study, conducted in one of the oldest and largest cosmopolitan treatment centers in Kenya, shows the significant HIV stigma, lack of HIV status disclosure and inadequate social support among the HIV infected patients is still noticeable. Further, HIV stigma, disclosure and social support contributes significantly nevirapine and efavirenz plasma concentrations by influencing ART drug adherence. Integrating socio-psychological support in the individualization of ART treatment could also lead to optimal ART plasma concentration.

\section{Declarations}

Funding: This study was supported by funds from KEMRI-Internal Grant (IRG/20) 2010/2011 and HIV Research Trust Scholarship (HIVRT13-091)

Conflicts of interest/Competing interests: None

Ethics approval: Ethical approval for this study was obtained from KEMRI Scientific Review Unit (SERU). The protocol number is SSC No. 2539

Consent to participate: Written informed consent was obtained from all subjects before the study.

Consent for publication: Was granted at the time of consenting

Page $11 / 15$ 
Availability of data and material: All data will be stored at figshare at the moment submitted as electronic data

Code availability: not applicable

Author's Contribution: MON, MO conceived the study. MON collected samples and conducted laboratory analysis. MO, WDB and FAO supervised laboratory analysis. MON analyzed the data and prepared the draft manuscript. MO, WDB and FAO provided guidance and mentorship during the implementation of the study. All authors reviewed and approved the final manuscript.

Acknowledgements: We would like to thank the study patients enrolled at the FACES- KEMRI HIV care and treatment program as well as the technical staff at KEMRI and the Retrovirology, Centre de Recherche Public de la Santé (CRPSanté), Luxembourg. We wish to acknowledge and Assistant Director CMR and the Director General KEMRI for allowing the publication of this work.

\section{References}

1. UNAIDS. 2020. Global AIDS update 2020. Seizing the Moment. Tackling entrenched Inequalities to end epidemics. Available https://www.unaids.org/sites/default/files/media_asset/2020_global-aids-report_en.pdf. Accessed August 16, 2020.

2. WHO. HIV/AIDS Psychosocial Support. Available at https://www.who.int/hiv/topics/psychosocial/support/en/\#references. Accessed March 7, 2021.

3. Okonji, E. F., Mukumbang, F. C., Orth, Z., Vickerman-Delport, S. A. \& Van Wyk, B. Psychosocial support interventions for improved adherence and retention in ART care for young people living with HIV (10-24 years): a scoping review. BMC Public Health, 20 (1), 1841 (2020).

4. Kose, S., Mandiracioglu, A., Mermut, G., Kaptan, E. \& Ozbel, Y. The Social and Health Problems of People Living with HIV/ AIDS in Izmir, Turkey. EAJM, 44, 32-39 (2012).

5. Mbonu, N. C., van den Borne, B. \& De Vries, N. Stigma of People with HIV/AIDS in Sub-Saharan Africa: A Literature Review. J Trop Med, 14, 1-14 (2009).

6. Greeff, M. et al. Disclosure of HIV status: experiences and perceptions of persons living with HIV/AIDS and nurses involved in their care in Africa. Qual Health Res, 18 (3), 311-324 (2008).

7. Rankin, W., Brennan, S., Schell, E., Laviwa, J. \& Rankin, S. The stigma of being HIV-positive in Africa. PLoS Medicine, 2, 8247 (2005).

8. Ministry of Health, National AIDS \& STI Control Program. Guidelines on Use of Antiretroviral Drugs for Treating and Preventing HIV Infection in Kenya 2018 Edition (NASCOP, Nairobi, Kenya, August 2018). Print

9. Veldkamp, A. I. et al. High exposure to nevirapine in plasma is associated with an improved virological response in HIV-1-infected individuals. AIDS, 15 (9), 1089-1095 (2001).

10. Mur, E., Droste, J. A., Bosch, M. \& Burger, D. M. Nevirapine plasma concentrations are still detectable after more than 2 weeks in the majority of women receiving single-dose nevirapine: implications for intervention studies. J Acquir Immune Defic Syndr, 39 (4), 419-421 (2005).

11. Pavlos, R. \& Phillips, E. J. Individualization of antiretroviral therapy. Pharmgenomics Pers Med, 5 (1), 1-17 (2012).

12. Ngayo, M. O. et al. Impact of First Line Antiretroviral Therapy on Clinical Outcomes Among HIV-1 Infected Adults Attending One of the Largest HIV Care and Treatment Program in Nairobi Kenya. J AIDS Clin Res, 7, 615 (2016).

13. Lemeshow, S., Hosmer, D. W., Klar, J. \& Lwanga, S. K. Adequacy of sample size in health studies (John Wiley \& Sons, 1990). 
14. Gunda, D. W. et al. Plasma Concentrations of Efavirenz and Nevirapine among HIV-Infected Patients with Immunological Failure Attending a Tertiary Hospital in North-Western Tanzania. PLoS ONE, 8 (9), e75118 (2013).

15. Oluka, M. N., Okalebo, F. A., Guantai, A. N., McClelland, R. \& Graham, S. M. Cytochrome P450 $2 B 6$ genetic variants are associated with plasma nevirapine levels and clinical response in HIV-1 infected Kenyan women: a prospective cohort study. AIDS Res Ther, 12, 10 (2015).

16. Reddy, S. et al. A LC-MS/MS method with column coupling technique for simultaneous estimation of lamivudine, zidovudine, and nevirapine in human plasma. J Anal Sci Technol, 7, 17 (2016).

17. Duong, M. et al. Usefulness of therapeutic drug monitoring of antiretrovirals in routine clinical practice. HIV Clin Trials, 5 (4), 216-223 (2004).

18. Gopalan, B. P. et al. Sub-therapeutic nevirapine concentration during antiretroviral treatment initiation among children living with HIV: Implications for therapeutic drug monitoring. PLoS One. 2017 Aug 21;12(8):e0183080

19. UNAIDS (2016) 'Prevention Gap Report'. Available at http://www.unaids.org/sites/default/files/media_asset/2016prevention-gap-report_en.pdf. Accessed January, 2021.

20. Phillips, E. J. \& Mallal, S. A. Personalizing antiretroviral therapy: Is it a reality? Per Med, 6 (4), 393-408 (2009).

21. Goffman, E. Stigma: Notes on the Management of Spoiled Identity (Simon and Schuster, 2009).

22. Ochieng, W. et al. Implementation and Operational Research: Correlates of Adherence and Treatment Failure Among Kenyan Patients on Long-term Highly Active Antiretroviral Therapy. J Acquir Immune Defic Syndr, 69 (2), e 49-56 (2015).

23. Gonzalez, D. et al. Nevirapine plasma exposure affects both durability of viral suppression and selection of nevirapine primary resistance mutations in a clinical setting. Antimicrob Agents Chemother, 49, 3966-3969 (2005).

24. Stutterheim, S. et al. Psychological and social correlates of HIV status disclosure: the significance of stigma Visibility. AIDS Educ Prev, 23 (4), 382-392 (2011).

25. Chambers, L. et al. Stigma, HIV and health: a qualitative synthesis. BMC Public Health, 15, 1-17 (2015).

26. Sears, B. HIV discrimination in health care services in Los Angeles County: the results of three testing studies. Wash Lee J Civ Rts Soc Just, 15, 85 (2008).

27. Rueda, S. et al. Examining the associations between HIV- related stigma and health outcomes in people living with HIV/AIDS: a series of meta-analyses. BMJ Open, 6, e011453 (2016).

28. Rao, D. et al. A structural equation model of HIV-related stigma, depressive symptoms, and medication adherence. AIDS Behav, 16, 711-716 (2012).

29. Sirikum, C. et al. HIV disclosure and its effect on treatment outcomes in perinatal HIV-infected Thai children. AIDS Care, 26 (9), 1144-1149 (2014).

30. Medley, A., Garcia-Moreno, C., McGill, S. \& Maman, S. Rates, barriers and outcomes of HIV serostatus disclosure among women in developing countries: implications for prevention of mother-to-child transmission programmes. Bull World Health Organ, 82, 299-307 (2004).

31. Johns, D. M., Bayer, R. \& Fairchild, A. L. Evidence and the Politics of Deimplementation: The Rise and Decline of the "Counseling and Testing" Paradigm for HIV Prevention at the US Centers for Disease Control and Prevention. Milbank Q, 94 (1), 126-162 (2016).

32. Brittain, K. et al. Social Support, Stigma and Antenatal Depression Among HIV-Infected Pregnant Women in South Africa. AIDS Behav, 21, 274-282 (2017).

33. Campbell, C., Yugi, N. \& Sbongile, M. Building contexts that support effective community responses to HIV/AIDS: A South African case study. Am J Community Psychol, 39 (3-4), 347-363 (2007). 
34. Zachariah, R. et al. Community support is associated with better antiretroviral treatment outcomes in a resourcelimited rural district in Malawi. Trans R Soc Trop Med Hyg, 101 (1), 79-84 (2007).

35. Handa, S., Halpern, C. T., Pettifor, A. \& Thirumurthy, H. The government of Kenya's cash transfer program reduces the risk of sexual debut among young people age 15-25. PLOS ONE, 9 (1), (2014). e85473

36. Cluver, L. et al. Achieving equity in HIV-treatment outcomes: can social protection improve adolescent ARTadherence in South Africa? AIDS Car, 28 (sup2), 73-82 (2016).

37. Bhana, A. et al. egt al. The VUKA family program: Piloting a family-based psychosocial intervention to promote health and mental health among HIV infected early adolescents in South Africa. AIDS Care - Psych Socio-Medical Aspects of AIDS/HIV 2014, 26(1), 1-11.

38. Kilburn, K., Thirumurthy, H., Halpern, C. T., Pettifor, A. \& Handa, S. Effects of a large-scale unconditional cash transfer program on mental health outcomes of young people in Kenya. J Adoles Health, 58 (2), 223-229 (2016).

39. Pettifor, A., Rosenberg, N. \& Bekker, L. G. Can cash help eliminate mother-to-child HIV transmission? Lancet HIV, 3 (2), e60-62 (2016).

40. UNAIDS. HIV and social protection guidance note. Retrieved from Geneva. 2014. Available at https://www.unaids.org/sites/default/files/media_asset/2014unaidsguidancenote_HIVandsocialprotection_en.pdf. Accessed March 8, 2021.

41. PEPFAR. (2015). Preventing HIV in adolescent girls and young women: Guidance for PEPFAR country teams on the DREAMS partnership. Washington, DC: USAID-PEPFAR. Available at http://childrenandaids.org/sites/default/files/2018-

11/Preventing\%20HIV\%20in\%20adolescent\%20girls\%20and\%20young\%20women\%20\%20Guidance\%20for\%20PEPFAR\%20country\%20teams\%20on\%20the\%20DREAMS\%20Partnership.pdf. Accessed February 2, 2021.

\section{Figures}

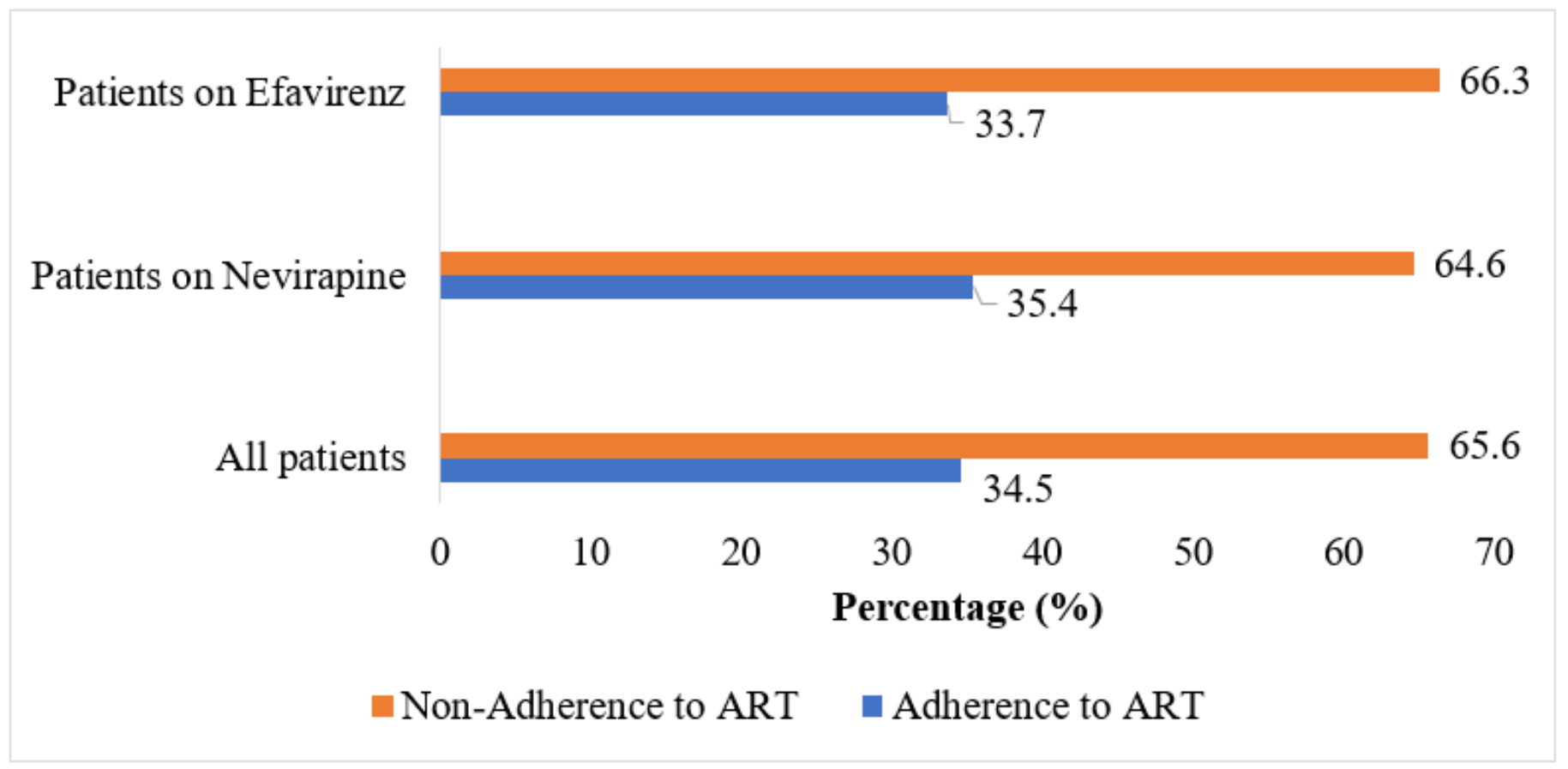

Figure 1 
Distribution of patients ART adherences in the past 30 days

\section{Supplementary Files}

This is a list of supplementary files associated with this preprint. Click to download.

- MusaStigmaData.xlsx 